\title{
CONSUMO DE BEBIDAS ALCOÓLICAS DURANTE A GRAVIDEZ
}

\author{
Emília Coutinho \\ Escola Superior de Saúde de Viseu, Instituto Politécnico de Viseu. CI\&DETS, Portugal \\ Carlos Pereira \\ Escola Superior de Saúde de Viseu, Instituto Politécnico de Viseu. CI\&DETS, Portugal \\ Odete Amaral \\ Escola Superior de Saúde de Viseu, Instituto Politécnico de Viseu. CI\&DETS, Portugal \\ Paula Nelas \\ Escola Superior de Saúde de Viseu, Instituto Politécnico de Viseu. CI\&DETS, Portugal \\ Cláudia Chaves \\ Escola Superior de Saúde de Viseu, Instituto Politécnico de Viseu. CI\&DETS, Portugal \\ Nélio Veiga \\ Health Sciences Institute - Universidade Católica Portuguesa Viseu, Portugal \\ Carla Cruz \\ Escola Superior de Saúde de Viseu, Instituto Politécnico de Viseu. CI\&DETS, Portugal
}

https://doi.org/10.17060/ijodaep.2017.n1.v3.988

Fecha de Recepción: 24 Enero 2017

Fecha de Admisión: 1 Abril 2017

\section{RESUMO}

Introdução: 0 consumo de bebidas alcoólicas durante a gravidez é altamente prejudicial à saúde da mãe bem como à saúde do feto. 0 consumo de álcool durante a gravidez leva-nos a questionar a qualidade dos cuidados de saúde. Os objetivos deste estudo foram quantificar a prevalência do consumo de bebidas alcoólicas durante a gravidez por mulheres portuguesas e imigrantes em Portugal e analisar a informação veiculada nas consultas de vigilância de gravidez.

Métodos: Estudo transversal, parcialmente analisado como caso controlo, envolveu 753 mães imigrantes com idades entre os 16 e os 45 anos $(29,30 \pm 5,76)$ e 1654 portuguesas com idades entre os 15 e os 44 anos $(29,99 \pm 5,42)$. Recolha de dados por questionário autopreenchido, em 32 maternidades/hospitais portugueses, de janeiro de 2010 a dezembro de 2011. Considerou-se consumo de bebidas alcoólicas a ingestão de qualquer quantidade de álcool durante a gravidez. As prevalências foram expressas em proporções e determinou-se ainda a magnitude de associação entre as variáveis com recurso aos Odds Ratio (OR) e respetivos intervalos de confiança a 95\%.

Resultados: Cerca de 9 em cada 10 das grávidas não ingeriu bebidas alcoólicas, mas entre as que ingeriram, a maior percentagem ocorreu entre as imigrantes (12,0\% vs 6,6\%) com significân- 


\section{CONSUMO DE BEBIDAS ALCOÓLICAS DURANTE A GRAVIDEZ}

cia estatística (OR 0,52; IC95\% 0,37-0,7; x2=19,617; $p<0,001$ ). Quanto à frequência do consumo, consumiram algumas vezes $57,8 \%$ das portuguesas e $71,1 \%$ das imigrantes (OR 0,37; IC95\% 0,26$0,52)$. Quanto à qualidade de informação sobre 0 consumo de álcool 39,2\% das portuguesas considerou-a boa e $34,9 \%$ das imigrantes, razoável.

Conclusão: As imigrantes consomem mais bebidas alcoólicas durante a gravidez que as portuguesas. A prevalência de qualquer consumo de álcool durante a gravidez por imigrantes e portuguesas sugere cuidados pré-natais inadequados.

Palavras Chave: álcool; gravidez; enfermeiro, educação para a saúde

\section{ABSTRACT}

\section{Consumption of alcoholic beverages during pregnancy}

Introduction: The consumption of alcoholic beverages during pregnancy is highly detrimental to the mothers' health as well as to the health of the fetus. Alcohol consumption during pregnancy leads us to question the quality of health care. The objectives of this study are to quantify the prevalence of alcohol consumption during pregnancy by Portuguese and immigrant women in Portugal and to analyze the information conveyed during pregnancy surveillance visits.

Methods: A cross-sectional study, partially analyzed as a control case, involved 753 immigrant mothers between the ages of 16 and $45(29,30 \pm 5,76)$ and 1654 Portuguese women aged 15-44 $(29,99 \pm 5,42)$. Data collection resorted to self-administered questionnaires in 32 Portuguese maternity wards/ hospitals, from January 2010 to December 2011. Alcohol consumption was defined as the intake of any amount of alcohol during pregnancy. Prevalence was expressed in proportions and the association scale between odds ratios (ORs) and their 95\% confidence intervals was determined.

Results: Nine out of ten pregnant women did not ingest any alcohol. Among those who ingested it, the highest percentage occurred among immigrants (12.0\% vs $6.6 \%)$ with statistical significance (OR 0.52, $\mathrm{Cl} 95 \% 0.37-0.7, \times 2=19.617, \mathrm{p}<0.001)$. As to the frequency of consumption, $57.8 \%$ of the Portuguese women and $71.1 \%$ of the immigrants (OR $0.37,95 \% \mathrm{Cl} 0.26-0.52$ ) consumed a few times. Regarding the quality of information on alcohol consumption, $39.2 \%$ of the Portuguese considered it good and $34.9 \%$ of the immigrants, reasonable.

Conclusion: Migrant women consume more alcoholic beverages during pregnancy than Portuguese ones. The prevalence of alcohol consumption during pregnancy by immigrants and Portuguese suggests inadequate antenatal care.

Keywords: Alcohol; pregnancy; Nurse, health education

\section{INTRODUÇÃO}

Muito se tem escrito e investigado sobre os efeitos do álcool na saúde das populações Em função da etapa de desenvolvimento humano, o seu consumo pode acarretar consequências mais ou menos nefastas e socialmente preocupantes. Muita da investigação realizada tem incidido na faixa etária dos adolescentes. Neste contexto, Ferreira e Torgal $(2011$, p.595) verificaram que são os adolescentes que consomem bebidas alcoólicas que mais referem ter tido relações sexuais, dando a entender que 0 álcool é utilizado pelos jovens como forma de facilitar o envolvimento sexual. Por sua vez a FCG (2013) alerta para o uso de álcool como sendo uma das causas de morte evitáveis; que esse consumo está a aumentar na população portuguesa; e que, de entre as pessoas com $15 \mathrm{e}$ mais anos, o consumo médio dos países da OCDE em litros per capita é de 9,1 e em Portugal é de 12,2 0 penúltimo pior, logo a seguir a França $(12,3)$. Em contrapartida, o relatório ESPAD (2015) sobre 0 consumo de álcool por jovens em Portugal e na Europa, regista que $71 \%$ dos jovens já consumiram bebidas alcoólicas sendo 73\% rapazes e 70\% raparigas; $42 \%$ consumiram bebidas alcoó- 
licas nos últimos 30 dias sendo 43\% rapazes e 41\% raparigas; e 9\% consumiram bebidas alcoólicas até atingir valores de intoxicação alcoólica valor igual em rapazes e raparigas. É neste enquadramento que chegamos à idade fértil e por sua vez passamos a manifestar a preocupação com 0 consumo de bebidas alcoólicas durante a gravidez. Neste sentido à que referir que o consumo de álcool, bem como 0 de outras drogas, pode acarretar consequências de gravidade extrema à vida e saúde humanas. Essa gravidade assume diferentes proporções em função da idade gestacional em que 0 consumo ocorre. Assentes em diferentes estudos sobre o consumo de álcool ao longo da gestação, Mello, Barrias e Breda (2001) bem como Carson et al. (2010) recordam que as consequências da exposição ao consumo de álcool no período pré-natal podem manifestar-se não apenas no feto mas ao longo de toda a vida; a percentagem de crianças que manifestam alterações da capacidade cognitiva e de ajustamento social é superior em filhas de mães que bebem comparativamente às filhas de mães que não ingerem bebidas alcoólicas; não há garantias de que haja uma quantidade mínima de álcool não prejudicial durante a gravidez pelo que a abstinência é a escolha prudente para a mulher grávida ou que pretenda engravidar; há que desenvolver intervenções apropriadas dirigidas à família de mulheres grávidas com dependência problemática de álcool.

No contexto mundial, a Organização Mundial de Saúde (WHO, 2012) e no contexto português, o Plano Nacional de Saúde 2004-2010 (ACS, 2009) e o Programa Nacional para a Vigilância da Gravidez de Baixo Risco (Portugal, DGS, 2015) salientam que está demonstrado que, comportamentos aditivos, como é o consumo de álcool durante a gravidez, se repercute negativamente na mãe e no feto/recém-nascido estando relacionadas com 0 aumento da probabilidade de mortalidade e morbilidade materna e infantil.

As diferentes culturas são tendencialmente protetoras da maternidade e impõem-se um conjunto de prescrições e interdições (Coutinho, 2015) que balizam formas de estar e de agir, que consideram seguras, em prol da saúde e do bem-estar da mãe e do feto. No entanto, o contexto migratório é habitualmente um contexto de vulnerabilidade. Em geral, os motivos que levam à migração (Coutinho et al, 2012), são contextos de eventual precariedade financeira, de desenraizamento familiar e ausência de uma rede de amigos. A dificuldade em conhecer e se adaptar a uma nova cultura podem ser geradores de ansiedade e stress podem encontrar eco numa cerveja ao fim do dia ou num copo de vinho à refeição.

Considera-se que a ingestão de álcool durante a gestação deve levar-nos a questionar o sistema de saúde e a qualidade dos cuidados pré-natais oferecidos. 0 sistema de saúde português prevê a gratuitidade da vigilância pré-natal incluindo consultas e exames pré-natais, para mulheres imigrantes e portuguesas como garante da qualidade dos cuidados que presta e como forma de assegurar a saúde, bem-estar e qualidade de vida das populações. A temática do consumo de álcool durante a gravidez faz parte dos programas de vigilância de saúde pré-natal sendo assegurada pelo Serviço Nacional de Saúde no âmbito dos cuidados de saúde primários.

Os objetivos deste estudo foram quantificar a prevalência do consumo de bebidas alcoólicas durante a gravidez por mulheres portuguesas e imigrantes em Portugal e analisar a informação veiculada nas consultas de vigilância de gravidez.

\section{PARTICIPANTES E MÉTODOS}

Estudo quantitativo, transversal, parcialmente analisado como caso-controlo, com uma amostra não probabilística por conveniência, que envolveu 753 imigrantes com idades compreendidas entre os 16 e os 45 anos $(29,30 \pm 5,76)$ e 1654 portuguesas com idades compreendidas entre os 15 e os 44 anos $(29,99 \pm 5,42)$.

A recolha de dados decorreu em 32 maternidades/hospitais, dos 18 distritos de Portugal 


\section{CONSUMO DE BEBIDAS ALCOÓLICAS DURANTE A GRAVIDEZ}

Continental, entre janeiro de 2010 e dezembro de 2011. Para a recolha de dados sociodemográficos, obstétricos e de vigilância de gravidez utilizámos um questionário autopreenchido.

Para quantificar o consumo de bebidas alcoólicas durante a gravidez considerou-se não haver consumo de bebidas alcoólicas quando a grávida não ingeriu nenhuma bebida alcoólica durante a gravidez, considerou-se consumo de bebidas alcoólicas quando a grávida ingeriu bebidas alcoólicas durante a gravidez, independentemente da quantidade e da frequência. Nas grávidas que ingeriram bebidas alcoólicas durante a gravidez considerou-se a frequência da ingestão em "Sim todos os dias", e "Sim algumas vezes".

Para analisar a informação fornecida sobre álcool durante as consultas de vigilância da gravidez considerou-se o profissional que forneceu a informação (médico e enfermeiro) e a qualidade da informação fornecida, sendo que para tal foram criados três grupos de coorte: qualidade de informação boa, razoável e má.

Recorreu-se ao programa estatístico SPSS 22.0 de apoio à análise estatística.

As prevalências foram expressas em proporções e comparadas pelo teste do qui-quadrado, com aplicação de um nível de significância de 0,05. Determinou-se ainda a magnitude de associação entre as variáveis com recurso aos Odds Ratio (OR) e respetivos intervalos de confiança a 95\% (IC95\%).

0 protocolo de recolha de dados foi aprovado pela Comissão Nacional de Proteção de Dados e pelas Comissões de Ética de todas as instituições envolvidas. A participação no estudo foi voluntária tendo sido obtido o consentimento informado das participantes e assegurada a confidencialidade dos dados tanto na recolha quanto no tratamento estatístico realizado.

\section{RESULTADOS}

Os resultados serão primeiramente apresentados em termos da prevalência de consumo de bebidas alcoólicas e por último em termos da educação para a saúde sobre 0 consumo de bebidas alcoólicas, realizada nas consultas de vigilância da gravidez

\section{Prevalência de consumo de bebidas alcoólicas}

Procurou-se saber os hábitos das grávidas relativamente ao consumo de bebidas alcoólicas. Tal como resultados insertos na tabela 1, é de referir que $8,3 \%$ da totalidade da amostra mencionou ter ingerido bebidas alcoólicas durante a gravidez e entre as que ingeriram, a maior percentagem recai sobre as imigrantes $(12,0 \%$ vs 6,6\%) com significância estatística ( $x 2=19,617 ; p<0,001)$.

Quanto à frequência do consumo de bebidas alcoólicas, $57,8 \%$ das portuguesas consumiram algumas vezes e $42,2 \%$ todos os dias. Nas imigrantes, $28,9 \%$ consumiram todos os dias e $71,1 \%$ algumas vezes. Os valores de Odds rátio e dos intervalos de confiança são significativos no que se refere ao sim, algumas vezes, como se pode verificar pelos valores apresentados na mesma tabela. 
Tabela 1

Consumo de bebidas alcoólicas e frequência de consumo durante a gravidez

\begin{tabular}{c|c|c|c|c|c|c|c|c}
\hline \multirow{2}{*}{$\begin{array}{c}\text { Ingestão de bebidas } \\
\text { alcoólicas }\end{array}$} & \multicolumn{2}{|c|}{ Portuguesas } & \multicolumn{2}{|c|}{ Imigrantes } & \multicolumn{2}{|c|}{ Total } & OR & IC95\% \\
\cline { 2 - 10 } & $\mathbf{n}$ & $\%$ & $\mathbf{n}$ & $\%$ & $\mathbf{n}$ & $\%$ & & \\
\hline Não & 1545 & 93,4 & 663 & 88,0 & 2208 & 91,7 & $1 *$ & \\
\hline Sim & 109 & 6,6 & 90 & 12,0 & 199 & 8,3 & 0,52 & $0,39-0,70$ \\
\hline \multirow{2}{*}{ Sim Todos os dias } & 46 & 42,2 & 26 & 28,9 & 72 & 36,2 & 0,76 & $0,47-1,24$ \\
\hline \multirow{2}{*}{ Sim Algumas vezes } & 63 & 57,8 & 74 & 71,1 & 127 & 63,8 & 0,37 & $0,26-0,52$ \\
\hline
\end{tabular}

\section{Educação para a saúde}

A educação para a saúde, durante a gravidez, é uma atividade prioritária por parte dos profissionais de saúde e, mais concretamente pelos enfermeiros, no sentido não só de promoverem estiIos de vida saudável, nomeadamente sem consumo de bebidas alcoólicas, e identificarem fatores de risco gravídico o mais precocemente possível, mas também o de promoverem a vivência em segurança de uma gravidez saudável e sem intercorrências para a mulher/casal e criança. Salienta-se que $90,1 \%$ das mulheres referem que Ihes foi abordada a temática do álcool, pelos profissionais de saúde, nas consultas de vigilância da gravidez.

Os dados apresentados na tabela 2, respeitam à informação sobre a temática "álcool" fornecida pelo médico e pelo enfermeiro, nas consultas em que ambos asseguraram cuidados de vigilância de saúde, sendo que o enfermeiro foi o profissional de saúde que mais informou sobre o consumo de álcool quer às grávidas portuguesas, quer às imigrantes (39,2\% vs $26,5 \%$ respetivamente).

Tabela 2

Informação prestada pelo enfermeiro e pelo médico sobre consumo de bebidas alcoólicas

\begin{tabular}{c|c|c|c|c|c|c|c|c}
\hline \multirow{2}{*}{$\begin{array}{c}\text { Profissional que informou } \\
\text { sobre álcool na gravidez }\end{array}$} & \multicolumn{2}{|c|}{ Portuguesa } & \multicolumn{2}{|c|}{ Imigrante } & \multicolumn{2}{|c|}{ Total } & \multirow{2}{*}{ X2 } & \multirow{2}{*}{$\mathbf{p}$} \\
\cline { 2 - 7 } & $\mathbf{n}$ & $\mathbf{\%}$ & $\mathbf{n}$ & $\mathbf{\%}$ & $\mathbf{n}$ & $\mathbf{\%}$ & & \\
\hline \multirow{2}{*}{ Médico } & 205 & 24,8 & 78 & 9,4 & 283 & 34,3 & & \\
\cline { 2 - 7 } Enfermeiro & 324 & 39,2 & 219 & 26,5 & 543 & 65,7 & & \\
\hline
\end{tabular}

Quanto à qualidade de informação $39,2 \%$ das portuguesas considerou-a boa e $34,9 \%$ das imigrantes considerou-a razoável.

\section{DISCUSSÃO}

No presente estudo quantificámos a prevalência do consumo de bebidas alcoólicas durante a 


\section{CONSUMO DE BEBIDAS ALCOÓLICAS DURANTE A GRAVIDEZ}

gravidez por mulheres portuguesas e imigrantes em Portugal e analisámos a informação veiculada nas consultas de vigilância de gravidez.

Deparámo-nos que cerca de uma em cada dez grávidas ingeriram bebidas alcoólicas durante a gravidez, sendo maior o valor entre as imigrantes. No contexto europeu, o estudo de Mardby, Lupattelli, Hensing, \& Nordeng (2017) refere que 15,8\% de uma amostra de 7905 mulheres consumiram álcool durante a gravidez, e alerta para a necessidade que a Europa una esforços no desenvolvimento de uma estratégia europeia comum para prevenir o consumo de álcool durante a gravidez. Também Baron et al., 2015) referem que 11,0\% da amostra, correspondendo a 659 mulheres grávidas, consumiram bebidas alcoólicas pelo menos uma vez durante a gravidez e que o consumo de álcool durante a gravidez foi associado a resultados negativos da gravidez. Enfatizam a necessidade de se repensar estratégias mais aperfeiçoadas para dar resposta às especificidades dos diferentes grupos sociodemográficos e culturais nomeadamente os de maior risco em que as desigualdades sociais sejam evidentes. Por sua vez Silva (2013) conclui que $36,2 \%$ das mulheres inquiridas ingeriu bebidas alcoólicas durante a gravidez. Sugere que se continue a investigação nesta área agora numa abordagem qualitativa de modo a compreender os motivos que levam as mulheres a continuar a ingerir bebidas alcoólicas durante a gravidez. Walker, Al-Sahab, Islam, \& Tamim, (2011) verificaram que 10,8\% das mulheres consumiram bebidas alcoólicas em algum momento da gravidez e que esse consumo estava associado a fatores como gravidezes indesejadas, tabagismo, entre outros. 0 status de imigrante revelou-se protetor do consumo de álcool durante a gravidez. Korinek e Smith (2011) verificaram que o consumo de álcool, era preditor de vigilância inadequada de gravidez (álcool OR=1,54 p<0,05). Oulman, Kim, Yunis, \& Tamim (2015) observaram uma maior probabilidade de ocorrência de gravidezes indesejadas nas mulheres que consumiram bebidas alcoólicas antes de engravidarem.

Quanto ao status imigrante contrariamente aos valores que nós determinámos, Tsai et al. (2017) verificaram que 0 consumo de bebidas alcoólicas durante a gravidez pelas mulheres nascidas nos EUA foi de $9 \%$ enquanto 0 consumo de bebidas alcoólicas durante a gravidez pelas mulheres imigrantes foi de $2 \%$, verificando-se um efeito protetor.

Em Portugal, Topa (2013) alerta para a assimetria nas relações de poder interprofissionais de saúde e as grávidas imigrantes, para a incompreensão e desconhecimento multicultural, para os discursos hegemónicos que se impõem e regulam os saberes e entendimentos das mulheres imigrantes. Fica-nos a questão: Em que medida este contexto condiciona a efetividade da educação para a saúde, nomeadamente a relativa ao consumo de bebidas alcoólicas durante a gravidez?

Rodrigues (2015) alerta para o facto de que as mulheres avaliaram a informação sobre o consumo de álcool transmitida em contextos de saúde durante a gravidez como bastante superficial e lata. Várias têm sido as tentativas de ultrapassar barreiras e promover a convivência cidadã, sendo o Alto Comissariado para as Migrações um exemplo de construção de pontes e de aproximação dos povos nomeadamente através do projeto Mediadores Interculturais nos Serviços Públicos e da Rede de Ensino Superior para a Mediação Intercultural entre outros, com os quais também se pretendeu e continua a pretender facilitar 0 acesso aos cuidados de saúde pelas populações imigrantes e assegurar cuidados de qualidade e sensíveis à diversidade cultural pelas mulheres grávidas imigrantes.

Yenal \& Sevil, 2012 recorreram à web para fazer educação e aconselhamento a mulheres grávidas tendo em conta as suas atividades de vida diária, e dos seus resultados, concluíram que esta também pode ser uma forma de fazer educação para a saúde por parte das enfermeiras e parteiras. Por sua vez Munro et al. (2017 exploraram uma aplicação "SmartMom Text Messaging for Prenatal Education" para promover estilos de vida saudáveis, nomeadamente o não consumo de bebidas alcoólicas durante a gravidez, no sentido de ir de encontro às necessidades e interesses das grávi- 
das em termos de informação. De acordo com Shimizu e Lima (2009) as grávidas que realizam a vigilância pré-natal com enfermeiros, declaram-se satisfeitas com as consultas, devido à forma como se estabelecem as relações de comunicação, na qual o acolhimento e a escuta são privilegiados. A consulta de enfermagem assume-se como um instrumento de suma importância, pois tem como finalidade garantir a extensão da cobertura e melhoria da qualidade pré- natal, principalmente por meio da introdução das ações preventivas e promocionais às gestantes. É requerido, do profissional além de competência técnico-científica, sensibilidade para compreender a cliente e o seu modo de vida mas também habilidade de comunicação, baseada na escuta e na ação dialógica (Shimizu \& Lima, 2009; Yenal \& Sevil, 2012).

\section{CONCLUSÃO}

Em conclusão, a prevalência de mulheres que ingeriram bebidas alcoólicas durante a gravidez foi de $8,3 \%$. (12,0\% nas imigrantes e $6,6 \%$ nas portuguesas) 0 enfermeiro foi o profissional de saúde que mais promoveu comportamentos saudáveis nomeadamente no que se refere à não ingestão de bebidas alcoólicas. Quanto à qualidade de informação $39,2 \%$ das portuguesas considerou-a boa e $34,9 \%$ das imigrantes considerou-a razoável. Sugere-se a realização de outros estudos, até de âmbito qualitativo, que procurem compreender o que leva a que as grávidas consumam bebidas alcoólicas durante a gravidez que permitam desenvolver intervenções efetivas no que à prevenção do consumo de álcool durante a gravidez diz respeito.

\section{AGRADECIMENTOS}

Este trabalho é financiado por fundos nacionais através da FCT - Fundação para a Ciência e Tecnologia, I.P., no âmbito do projeto UID / Multi / 04016/2016. Além disso, gostaríamos de agradecer ao Instituto Politécnico de Viseu e ao CI\&DETS pelo seu apoio, e ao Alto Comissariado para as Migrações através da Rede de Ensino Superior em Mediação Intercultural.

\section{REFERÊNCIAS BIBLIOGRÁFICAS}

A. C. S. (2009). Plano Nacional de Saúde 2004-2010 - Avaliação de Indicadores. Lisboa.

Baron, R., Manniën, J., Velde, S., Klomp, T., Hutton, E. K., \& Brug, J. (2015). Socio-demographic inequalities across a range of health status indicators and health behaviours among pregnant women in prenatal primary care: A cross-sectional study. BMC Pregnancy and Childbirth, 15, 261. doi: 10.1186/s12884-015-0676-Z

Carson, G., Cox, L. V., Crane, J., Croteau, P., Graves, L., Kluka, S., . . Wood, R. (2010). Alcohol use and pregnancy consensus clinical guidelines. J Obstet Gynaecol Can, 32(8 Suppl 3), S1-31.

Coutinho, E. (2015). Vivências e Experiências das Várias Populações Migrantes: Crenças, Desejos e Práticas Culturais Durante a Gravidez. In ESEL (Ed.), I Congresso Internacional de Saúde Materna/I International Congress of Maternal Health (Escola Superior de Enfermagem de Lisboa (ESEL) - Departamento de Enfermagem de Saúde Materna ed., pp. 7-16). Lisboa, ESEL Auditório do polo Artur Ravara: ESEL. Retrieved from http://www.esel.pt/NR/rdonlyres/2A5DECA7-E259-421F-A31E-E6C141D229C9 /0/ebook_ci_saude_materna.pdf.

Coutinho, E., Silva, A., Pereira, C., Duarte, J., Chaves, C., Parreira, V., . . Barreto, C. (2012). Pregnant women immigrants in Portugal: Why they left their country? Revista Iberoamericana de Salud y Ciudadanía, 1(1, Enero-Junio), 99-118.

ESPAD Group. (2016). ESPAD Report 2015: Results from the European school survey project o alcohol and other drugs. Luxembourg: Office of The European Union. Acedido em http://www.espad.org/sites/espad.org/files/ESPAD_report_2015.pdf 
Ferreira, M. M. S. R. S., Torgal, M. C. L. F. P. R. (2011). Estilos de vida na adolescência: Comportamento sexual dos adolescentes portugueses. Revista da Escola de Enfermagem da USP, 45(3), 589-595. Acedido em http://www.scielo.br/pdf/reeusp/v45n3/v45n3a06.pdf

Fundação Calouste Gulbenkian, \& Health Cluster Portugal. (2013). Health in Portugal: A challenge for the future, The Gulbenkian platform for a sustainable health system. Lisboa: FCG. Acedido em - $\quad$ https://gulbenkian.pt/wp-content/uploads/2016/03/PGIS BrochuraSumario ExecutivoHealthPortugues.pdf

Grinfeld, H. (2009). Consumo nocivo de álcool durante a gravidez. In Arthur Guerra de Andrade \& J. C. Anthony (Eds.), Álcool e suas conseqüências: Uma abordagem multiconceitual (pp. 179199). São Paulo: Editora Manole. Acedido em http://cisa.org.br/UserFiles/File/alcoolesuasconsequencias-pt-cap9.pdf

Harding, S, Santana, P., Cruickshank, J. K., \& Boroujerdi, M. (2006). Birth weights of black African babies of migrant and nonmigrant mothers compared with those of babies of European mothers in Portugal. Annals of Epidemiology, 16(7), 572-579. Acedido em http://www.sciencedirect.com/science/article/pii/S104727970500356X

Korinek, K., \& Smith, K. R. (2011). Prenatal care among immigrant and racial-ethnic minority women in a new immigrant destination: exploring the impact of immigrant legal status. Soc Sci Med, 72(10), 1695-1703. doi: 10.1016/j.socscimed.2011.02.046

Mårdbya, A-C., Lupattellic, A., Hensingb, G., \& Nordeng, H. (2017). Consumption of alcohol during pregnancy: A multinational European study. Women and Birth, (In Press). Acedido em http://www.sciencedirect.com/science/article/pii/S1871519217300057

Mello, M. L. M., Barrias, J. C., \& Breda, J. (2001). Álcool e problemas ligados ao álcool em Portugal. Lisboa: Direcção-Geral da Saúde.

Munro, S., Hui, A., Salmons, V., Solomon, C., Gemmell, E., Torabi, N., \& Janssen, P. A. (2017). SmartMom Text Messaging for Prenatal Education: A qualitative focus group study to explore Canadian women's perceptions. JMIR Public Health Surveill, 3(1), e7. Acedido em https://www.ncbi.nlm.nih.gov/pmc/articles/PMC5320393/pdf/publichealth v3i1e7.pdf

Oulman, E., Kim, T. H. M., Yunis, K., \& Tamim, H. (2015). Prevalence and predictors of unintended pregnancy among women: An analysis of the Canadian Maternity Experiences Survey. BMC Pregnancy Childbirth, 15, 260. doi 10.1186/s12884-015-0663-4

Pacheco, M. J. N. (2012). Visitação domiciliária à puérpera e ao recém-nascido do Concelho de Tavira (Dissertação de mestrado, Instituto Politécnico de Beja). Acedido em https://repositorio.ipbeja.pt/handle/123456789/3973

Portugal, Direção Geral da Saúde. (2015). Programa nacional para a vigilância da gravidez de baixo risco. Lisboa: DGS. Acedido em https://www.dgs.pt/em-destaque/programa-nacional-para-avigilancia-da-gravidez-de-baixo-risco.aspx

Rodrigues, A. P. C. (2015). Consumos de bebidas alcoólicas e falsos conceitos: Questões de género numa população que recorre aos serviços da Unidade de Cuidados de Saúde Personalizados em Peso da Régua (Dissertação de mestrado, Universidade Fernando Pessoa). Acedido em http://bdigital.ufp.pt/handle/10284/5210

Santana, P., Santos, R., Alves, I., Couceiro, L., \& Machado, M. C. (2015). Avaliação das condições individuais e contextuais no peso dos recém-nascidos. Revista Portuguesa de Saúde Pública, 33(2), 157-169. Acedido em http://www.sciencedirect.com/science/article/ pii/S0870902515000103

Shimizu, H., \& Lima, M. (2009). As dimensões do cuidado pré-natal na consulta de enfermagem. Revista brasileira de enfermagem, 62, 387-392. 
Silva, R. M. G. H. (2013). Consumo de álcool e atitudes sobre a gravidez e a maternidade num grupo de grávias utentes da unidade de saúde de Matosinhos (Dissertação de mestrado, Universidade Católica Portuguesa). Acedido em http://repositorio.ucp.pt/handle/10400.14/17089

Topa, J. B. (2013). Cuidados de saúde materno-infantis à população imigrante residente em Portugal (Tese de doutoramento, Universidade do Minho). Acedido em http://repositorium.sdum.uminho.pt/handle/1822/26676

Tsai, H.-J., Surkan, P. J., Yu, S. M., Caruso, D., Hong, X., Bartell, T. R., ... Wang, X. (2017). Differential effects of stress and African ancestry on preterm birth and related traits among US born and immigrant black mothers. Medicine (Baltimore), 96(5), e5899. doi: 10.1097/MD.0000000000005899

Walker, M. J., Al-Sahab, B., Islam, F., \& Tamim, H. (2011). The epidemiology of alcohol utilization during pregnancy: An analysis of the Canadian Maternity Experiences Survey (MES). BMC Pregnancy Childbirth, 11, 52. doi: 10.1186/1471-2393-11-52

World Health Organization. Meeting to Develop a Global Consensus on Preconception Care to Reduce Maternal and Childhood Mortality and Morbidity. [Online]. Geneva: WHO Library Cataloguing-in-Publication; 2012 [cited 2014 Fevereiro. Acessível em: http://apps.who.int/ iris/bitstream/10665/78067/1/9789241505000_eng.pdf.

Yenal, K., \& Sevil, Ü. (2012). The effects of web-based childbirth education program on activities of daily living of pregnant women. HealthMed, 6(7), 930-938. 
